\title{
VERA observation of the massive star forming region $\mathrm{G} 34.4+0.23$
}

\author{
T. Kurayama \\ Mizusawa VERA observatory, NAOJ, \\ Osawa 2-21-1, Mitaka, Tokyo, 181-8588, Japan \\ email : t.kurayama@nao.ac.jp
}

\begin{abstract}
We observed with VERA the massive star forming region G34.4+0.23, to obtain parallaxes and proper motions. Four infrared dark clouds were observed and water maser were found in two dark clouds, MM1 and MM4. In MM1, the distribution of maser spots shows a "Vshaped" structure and most features co-moving with this structure. Phase-referenced images have peaks and their motion is much larger than the expected parallax. Further analysis is needed to correctly interpret our measurement of parallax.
\end{abstract}

Keywords. stars: formation, ISM: individual (G34.4+0.23), ISM: kinematics and dynamics, astrometry

\section{Introduction}

Infrared Dark Clouds (IRDCs) are found in massive star forming regions. They are thought to be an early stage of star formation. One of them is in the region of G34.4+0.23. In this region, four IRDCs (MM1-MM4) are observed, all located in the north-south direction. The IRAS point source IRAS $18507+0121$ and a compact HII region are associated with MM2. Water maser was detected in this IRAS source (Scalise Rodíguez \& Mendza-Torres (1989)).

Rathmorne et al. (2005) derived the masses of IRDCs from absolute luminosities and blackbody temperatures. Absolute luminosities depend on the distances, so the distance information is very important. We can also observe the outflows manifesting as the motions of maser features.

\section{Observation}

Four stations of VERA were used to observe water masers at $22 \mathrm{GHz}=1.3 \mathrm{~cm}$. The phase-referencing technique was adopted for VLBI measurements of parallaxes and proper motions. The reference source for phase-referencing was J1855+0251. We have observed at six dates until June 2007 and the monitoring continues. At each date, we observed for nine hours. The observational dates were Oct 21 2006, Nov 16 2006, Jan. 04 2007, Feb 10 2007, Mar 252007 and May 42007.

\section{Results}

Figure 1a shows the cross-power spectra for MM1. The time-dependent components are found at 40 and $90 \mathrm{~km} / \mathrm{s}$. Since the systemic velocity is about $60 \mathrm{~km} / \mathrm{s}$, most components are blueshifted. Figure 1b shows cross-power spectra for MM4 after the phase-referencing. 

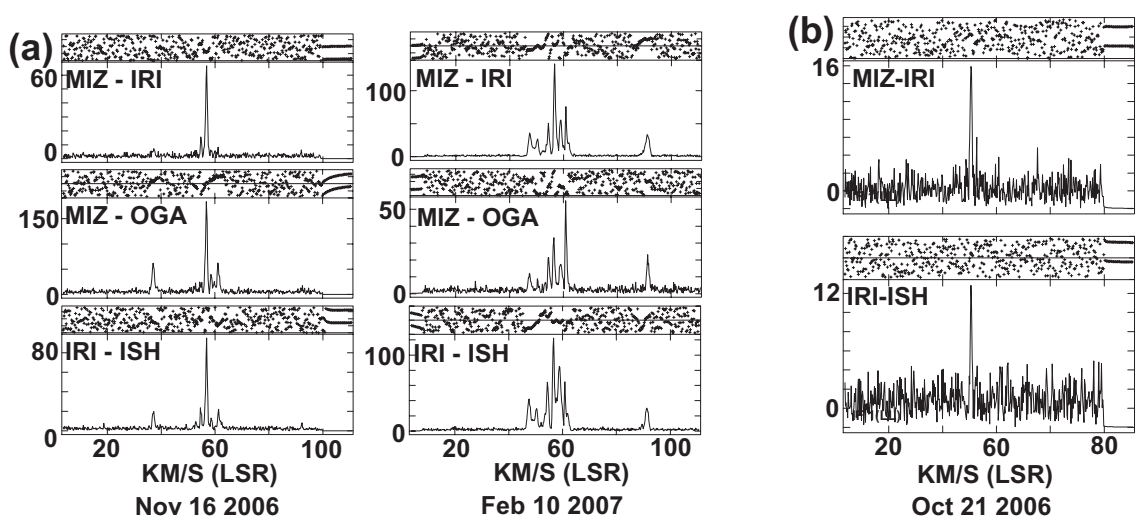

Figure 1. (a) Cross-power spectra for MM1. (b) Cross-power spectra for MM4. (b) is a phase-referenced spectra, while (a) is not.

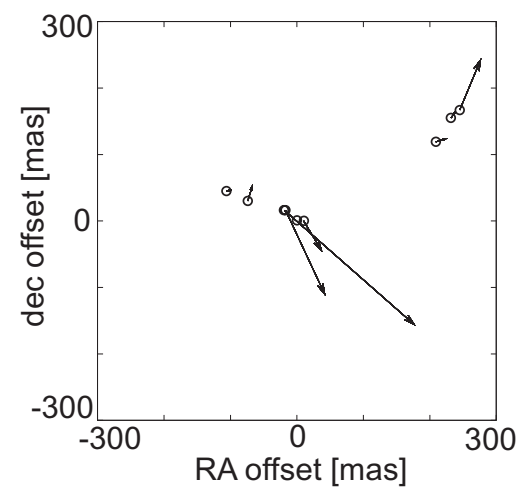

Figure 2. Distribution and linear motions of water masers in G34.4+0.23 between Oct 21 2006 and Feb 10 2007. Arrows represent the motion in 10 years.

Figure 2 shows the distribution and linear motions of maser features. They show a "Vshaped" structure and most of their linear motion is along this "V-shaped" structure.

\section{Measurement of parallax}

The phase-referenced images, the positions of which are referenced to the extragalactic source, have a few peaks. Their apparent motions are much larger than expected. Therefore, an additional analysis is needed to detect and quantify the true parallactic motion.

\section{References}

Rathmorne, J. M. et al. 2005, ApJ, 630, L181

Scalise, E. Jr., Rodríguez, L. F., \& Mendoza-Torres, E. 1989, A\&A, 221, 105 